\title{
Effekte körperlichen Trainings auf Lymphödeme bei Brustkrebs: Eine systematische Übersichtsarbeit
}

Baumann FT, Reike A, Reimer V et al. Effects of physical exercise on breast cancer-related secondary lymphedema: a systematic review. Breast cancer research and treatment 2018: 1-13

\section{Hintergrund}

Lymphödeme sind eine der häufigsten Nebenwirkungen, unter welchen Patientinnen mit Brustkrebs leiden [1]. Lymphödeme sind Flüssigkeitsansammlungen im Interstitium, die sich als Schwellungen äußern [2]. Die Ursache liegt in einer Schädigung des Lymphsystems, z.B. durch operative Entfernung von Lymphknoten, Bestrahlung oder tumor-induzierter Neo-Lymphangiogenese, der tumorbedingten Neubildung von Lymphgefäßen [2]. Aufgrund der Lokalisation des Tumors betreffen die Lymphödeme bei Patientinnen mit Brustkrebs die obere Extremität. Die typischen Symptome eines Lymphödems sind neben der deutlich sichtbaren Schwellung Gefühle von Schwäche und Schwere, kinästhetisches Unwohlsein sowie Bewegungseinschränkungen, welche bis zum Schmerz reichen können [1]. Zur Behandlung des Lymphödems wird normalerweise eine multimodale Therapie gewählt, bestehend aus Medikamenten, Physiotherapie (Lymphdrainage) und Hautpflege [3]. Hinsichtlich körperlicher Aktivität existieren noch keine klinischen Empfehlungen. Dementsprechend unsicher sind die Patienten auch hinsichtlich verschiedenster Alltagsaktivitäten, was letztlich in einer Reduzierung des körperlichen Aktivitätsniveaus mündet und zu einer Zunahme an Körpermasse führen kann, welche wiederum mit einem erhöhten Ödemrisiko assoziiert ist. Bis jetzt existieren eine Reihe von Übersichtsarbeiten, welche sich der Wirksamkeit von Krafttraining auf das Lymphödem annahmen. Die hier vorgestellte Arbeit von Baumann et al. ist aber die erste, welche sich auch anderer Bewegungsformen annimmt.

\section{Studienbeschreibung}

Von den 822 mit Hilfe eines Suchalgorithmuses in PubMed initial gefundenen Studien wurden letztlich 11 Studien mit sehr hoher Qualität, 9-mal höchstes Evidenzlevel-1a und 2-mal zweithöchstes Evidenzlevel-1b für Einzelstudien (American Cancer Society), in das systematische Review eingeschlossen. Alle Studien zusammengenommen, lagen Daten von insgesamt 458 Patientinnen vor, die Stichprobengröße der Einzelstudien rangiert zwischen 14 und 141 Patientinnen, deren mittleres Alter bei 56,8 Jahren liegt.

Gemessen wurde das Volumen des Lymphödems in den eingeschlossenen Studien auf 3 Weisen: der Wasserverdrängungsmethode, per nicht-invasivem optoelektronischem Scan oder anhand geometrischer Ableitungen aus mehreren Umfangsmessungen. Das Ausmaß des Lymphödems wurde durch die Volumendifferenz von operiertem und kontralateralem Arm der Patientinnen festgestellt.

\section{Interventionen}

In 4 der 11 Studien wurde ein Krafttraining durchgeführt, in 2 Studien wurde dieses mit Ausdauertraining kombiniert. 2 Arbeiten berichten über ein Training im Wasser, 1 Studie über Yoga, 1 Studie über Mobilisationen gegen die Schwerkraft und 1 Studie über eine Kombination aus Tai-Chi, QiGong und Mobilisationen gegen die Schwerkraft. Die Interventionsperioden reichten von Kurzzeitinterventionen mit 8 Wochen Dauer bis hin zu Interventionen, welche 1 Jahr umspannten. Die Trainingsfrequenz reichte von 1 wöchentlichen Trainingseinheit bis hin zu täglichem Training in dementsprechend kleinerem zeitlichem Umfang.

\section{Ergebnisse}

4 der 11 Studien berichten eine statistisch signifikante Minderung des Ödemvolumens und 6 Arbeiten eine statistisch signifikante subjektive Besserung. Als besonders positiv zu bewerten ist, dass keine Studie unerwünschte Ereignisse berichtete. Auch die Adhärenz zeigte sich extrem hoch mit nur wenigen bis gar keinen Drop-Outs. Was die Studien außerdem gemeinsam hatten, war eine wenigstens initiale Supervision des Trainings und eine hohe Individualisierung der Trainingsprogramme.

\section{Ausblick}

Ziel zukünftiger Forschung sollte sein, die Effekte der Interventionen zu konkretisieren und klare evidenzbasierte Empfehlungen für das bewegungstherapeutische Management von Lymphödemen zu entwickeln. Berücksichtigt werden muss zudem, dass beispielsweise die wassergestützte Therapie bei Patientinnen parallel zur Chemotherapie wegen der erhöhten Infektionsgefahr kontraindiziert ist. Zur effektiven Trainingssteuerung sollte in Trainingsstudien auch eine systematische Dokumentation der Trainingsvariablen stattfinden, sodass auch quantitative Vergleiche möglich werden. Insgesamt stärken die Ergebnisse das Vertrauen in die Breite der Bewegungstherapie.

\section{Kommentar zur Studie}

\section{WICHTIGES ARGUMENT!}

Gerade für den therapeutischen Alltag bietet die Arbeit von Baumann und Kollegen eine wichtige Argumentationsstütze. Aufgrund antiquierter Meinungen und der unüberschaubaren Verfügbarkeit von Informationen begegnet uns in der täglichen Arbeit mit Patientinnen mit Lymphödemen der oberen Extremität eine große 
Unsicherheit, die bis hin zur Bewegungsangst reicht. Die psychologische Wirkung mit Blick auf die Bewegungsförderung, welche mit der Aufklärung der Patientinnen einhergeht, darf hierbei keinesfalls unterschätzt werden.

Zwar war durch die 2009 hochrangig publizierte Arbeit von Schmitz et al. [4] ein Meilenstein geschaffen worden zur argumentativen Untermauerung der Unbedenklichkeit von Krafttraining bei Brustkrebspatientinnen mit Lymphödemen, allerdings beschränkten sich die Daten auf Widerstandstraining. Die Beleuchtung anderer Bewegungsformen, wie Baumann et al. sie vornahmen, war daher ein wichtiger nächster Schritt.

\section{Ein Blick zurück}

Wirft man einen Blick in die Historie bewegungstherapeutischer Forschung, schließt diese Arbeit an die Bemühungen der Kölner Arbeitsgruppe um Prof. Dr. Klaus Schüle von vor über 30 Jahren an. Bereits 1985 sprachen Schüle und Trimborn in „Rehabilitation nach Mammakarzinom“ Empfehlungen zum Umgang mit Bewegung bei Lymphödemen aus [5], welche aufgrund der damaligen Ermangelung an Studien vor allem auf Expertenmeinungen und therapeutischer Erfahrung fußten. Heute sagt Prof. Schüle:
„Was uns damals fehlte, waren zur Evaluation standardisierte Erhebungsbögen. Die kamen dann alle später. Insofern waren eben auch unsere ,Studien“ überwiegend Erfahrungsberichte, die zu ,wissenschaftlichen' Ergebnissen nicht ausreichten. Trotzdem denke ich, dass diese ,evidence based practice' sehr nützlich für die betroffenen Frauen war." Prof. Schüle erinnert sich, wie kontrovers das Thema in den 1980er-Jahren diskutiert wurde: „Einerseits gab es die medizinischen Expertisen, die einen vergleichsweise konservativen Standpunkt hatten und initial Schonung befürworteten, andererseits vertraten die bewegungstherapeutischen Professionen einen progressiveren Umgang, welcher eine möglichst zeitnahe Mobilisation forderte." Letzteres erschien nötig, insbesondere mit Blick auf den raschen Funktionsverlust der Schulter durch Immobilisation.

Es sei abschließend erwähnt, dass die Operationsmethoden in den letzten Jahrzehnten enorm an Qualität gewannen und heute deutlich sanfter sind, mit besonderem Blick auf die Erhaltung der Brust und schonenderem Vorgehen bei der axillären Lymphknotenentnahme. Dementsprechend sind sowohl die relative Inzidenz als auch der Schweregrad der Nebenwirkungen zurückgegangen.

\section{Autorinnen/Autoren}



Maximilian Köppel

Universität Heidelberg Institut für Sport und Sportwissenschaft Im Neuenheimer Feld 700

69120 Heidelberg Tel.: 0176-24023548

E-Mail: maximilian.koeppel@outlook.de

\section{Literatur}

[1] DiSipio T, Rye S, Newman B, Hayes S. Incidence of unilateral arm lymphoedema after breast cancer: a systematic review and meta-analysis. The lancet oncology 2013; 14: 500-515

[2] Mortimer P. Arm lymphoedema after breast cancer. The Lancet Oncology 2013; 14: 442-443

[3] Liebl ME, Preiß S, Pögel $S$ et al. Elastic tape as a therapeutic intervention in the maintenance phase of complex decongestive therapy (CDT) in lymphedema. Physikalische Medizin, Rehabilitationsmedizin, Kurortmedizin 2014; 24: 34-41

[4] Schmitz KH, Ahmed RL, Troxel A et al. Weight lifting in women with breast-cancerrelated lymphedema. New England Journal of Medicine 2009; 361: 664-673

[5] Schüle K, Trimborn S. Rehabilitation nach Mammakarzinom. München: Pflaum; 1985 